\title{
LINEAR AND NON-LINEAR PROBLEMS OF PLATE DYNAMICS
}

\author{
P. Baradokas, E. Michnevič, L. Syrus
}

Department of Theoretical Mechanics, Vilnius Gediminas Technical University, Sauletekio al. 11, LT-10223 Vilnius, Lithuania. E-mail: edmich@fm.vgtu.lt

Received 20 Apr 2007, accepted 10 Oct 2007
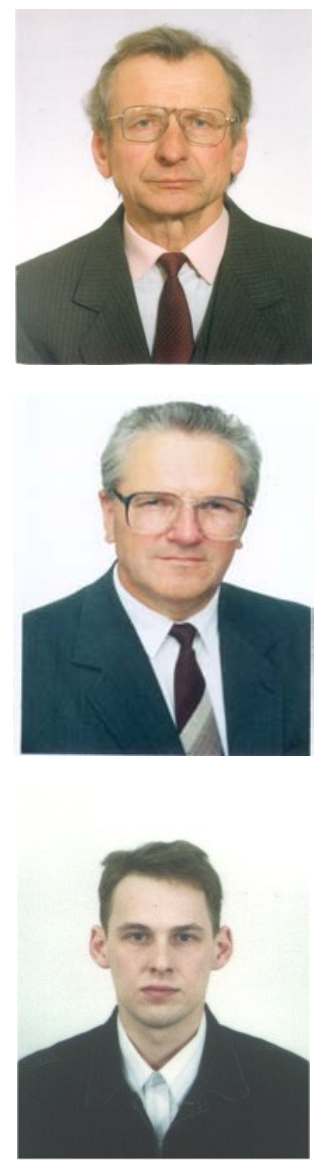

Petras BARADOKAS, Doc Assoc Prof

Education: In 1964 a graduate of Mechanics Technology engineering studies at Kaunas University of Technology (formerly Kaunas Polytechnic Institute).

Affiliations and functions: Doctor's degree in 1971. From 1975, Associate Professor.

Scientific interests: dynamics and optimization of composite structures.

Edvard MICHNEVISH, Doc Assoc Prof

Education: In 1993 a graduate of Mechanics Technology engineering studies at Vilnius Gediminas Technical

University. Doctor's degree in 2000.

Affiliations and functions: From 2001, Associate Professor.

Scientific interests: dynamics and optimization of composite structures.

Leonidas SYRUS, Doc Assoc Prof

Education: In 1959 a graduate of Mechanics Technology engineering studies at Kaunas University of Technology (formerly Kaunas Polytechnic Institute).

Affiliations and functions: Doctor's degree in 1973. From 1975 - Associate Professor.

Scientific interests: dynamics and optimization of composite structures.

Abstract. This paper presents a comparative analysis of linear and non-linear problems of plate dynamics. By expressing the internal friction coefficient of the material by power polynomial $\gamma=\gamma_{0}+\gamma_{1} \varepsilon_{0}+\gamma_{2} \varepsilon_{0}^{2}+\ldots$, we assume $\gamma=\gamma_{0}=$ const for a linear problem. When at least two polynomial terms are taken, a non-linear problem is obtained. The calculations of resonance amplitudes of a rectangular plate yielded 3 per cent error: a linear problem yields a higher resonance amplitude. Using the Ritz method and the theory of complex numbers made the calculations. Similar methods of calculation can be used in solving the dynamic problems of thin-walled vehicle structures.

Keywords: plates, linear analysis, nonlinear analysis.

\section{Introduction}

Most parts of a vehicle body consist of a light frame covered with thin-walled shells and plates. The dynamic loads that develop during operation cause the vibration of these structures. Though the dynamic problems of shells and plates have been discussed in many papers, some issues require special consideration [1, 2, 6, 8-11, 13].

The dissipation of oscillational energy in material is usually expressed by the logarithmic decrement $\delta\left(\varepsilon_{0}\right)$ or the internal friction coefficient $\gamma=\gamma\left(\varepsilon_{0}\right)$, in which $\varepsilon_{0}$ is the amplitude of deformation. The relationship between 
them can be expressed simply as $\delta=\pi \gamma$. As shown by experimentally obtained tables and graphs, this function is a monotonically rising curve. Rarely, $\delta\left(\varepsilon_{0}\right)=$ const or is a curve with several extremums [1].

Assuming $\delta\left(\varepsilon_{0}\right)=$ const, we get a linear problem and the calculations are simplified to a large extent. This allows relatively easy solution of a wide range of interesting problems [2, 6, 8, 9]. The error of linear analysis can be determined only by comparing the calculation results. The present paper aims to provide at least partial solution to this problem.

\section{Formulation of the problem}

The investigation is based on E. S. Sorokin's hypothesis, combined with some elements of non-linear analysis [14].

By applying methods of approximating functions, the experimentally determined relationship $\gamma=\gamma\left(\varepsilon_{0}\right)$ can be expressed by the power polynomial

$$
\gamma=\gamma_{0}+\gamma_{1} \varepsilon_{0}+\gamma_{2} \varepsilon_{0}^{2}+\ldots \text {, where } \gamma_{i} \text { are constants. }
$$

When one term, $\gamma=\gamma_{0}$, of the polynomial is taken, we will get a linear solution of linear analysis. When two or more terms are taken, a non-linear solution is obtained. The results yielded by the aforementioned two approaches are compared.

Forced oscillations of a rectangular plate are considered. A variation oscillation equation is obtained by applying Hamilton's principle. The Ritz method and a theory of complex numbers are used to solve the problem $[3-5,7]$.

\section{Solution of linear and non-linear problems}

Hamilton's principle applied to a system of forces, including inelastic forces, can be expressed by a variation equation as follows:

$$
d S=\delta \int_{\tau_{1}}^{\tau_{2}}[T-(1+i \gamma) V+W] d t=0
$$

in which $T$ and $V$ are kinetic and potential energy of the system, $W$ is the work of external forces, $\gamma$ is a damping coefficient, $\left(\tau_{2}-\tau_{1}\right)$ is a short time interval, and $t$ is time. By expressing the terms of the equation in a complex form, we get $T=T_{0} e^{2 i \omega t}, V=V_{0} e^{2 i \omega t}$, and $W=W_{0} e^{2 i \omega t}$. Since $e^{2 i \omega t} \neq 0$, we get the following expression from equation (1):

$$
\delta\left[T_{0}-(1+i \gamma) V_{0}+W_{0}\right]=0
$$

The solution to the equation can be in the form of three variables of the function:

$$
w(x, y, t)=w_{0}(x, y) e^{i \omega t}
$$

The function of the oscillation form $w_{0}(x, y)$ can be expressed as follows:

$$
w_{0}(x, y)=a_{1} \Phi_{1}(x, y)+a_{2} \Phi_{2}(x, y)+\ldots+a_{k} \Phi_{k}(x, y) .
$$

in which $\Phi_{k}(x, y)$ denotes the basic functions known.

In this way, equation (2) is replaced with an equation system of particular derivatives of the coefficients $a_{k}$ :

$$
\frac{\delta}{\delta a_{k}}\left[T_{0}-(1+i \gamma) V_{0}+W_{0}\right]=0
$$

in which $k=1,2, \ldots, n$.

In the case of a rectangular isotropic plate, when $\gamma=\gamma_{0}+\gamma_{1} \varepsilon_{0}$, we get

$$
\begin{gathered}
T_{0}=\mu h \omega^{2} \int_{0}^{a} \int_{0}^{b} W_{0} d x d y \\
(1+i \gamma) V_{0}=\frac{1}{2} \int_{-h}^{h} \int_{0}^{a} \int_{0}^{b}\left\{\sigma_{x} \varepsilon_{x}\left[1+i\left(\gamma_{0}+\gamma_{1} \varepsilon_{0 x}\right)\right]+\right. \\
+\sigma_{y} \varepsilon_{y}\left[1+i\left(\gamma_{0}+\gamma_{1} \varepsilon_{0 y}\right)\right]+ \\
\left.+\tau_{x y} \varepsilon_{x y}\left[1+i\left(\gamma_{0}+\gamma_{1} \varepsilon_{0 x y}\right)\right]\right\} d x d y d z \\
W_{0}=F_{0} W_{F}
\end{gathered}
$$

in which $\mu$ is the mass of the plate unit area; $2 h$ is plate thickness, $F_{0}$ is the amplitude of the excitation force, and $w_{F}$ is force point displacement.

Beam functions are chosen as the basic functions for a rectangular plate. Let us consider the first type of oscillation of the plate supported around the periphery. In this case, we have

$$
W_{0}(x, y)=a_{1} \sin \frac{\pi x}{a} \sin \frac{\pi y}{b}
$$

Substituting expression (7) in formula (6) and performing some mathematical operations, we get:

$$
\begin{aligned}
& T_{0}=\frac{\mu h \omega^{2} a b}{4} a_{1}^{2}, \\
& (1+i \gamma) V_{0}=\frac{\pi^{4} D K_{1}}{4} a_{1}^{2}+i \gamma_{0} \frac{\pi^{4} D K_{1}}{4} a_{1}^{2}+ \\
& \quad+i \gamma_{1} \frac{\pi^{4} D h K_{2}}{3} a_{1}^{3}, \\
& W_{0}=F_{0} a_{1},
\end{aligned}
$$


where $K_{1}=\frac{\left(a^{2}+b^{2}\right)^{2}}{a^{3} b^{3}}, K_{2}=\left(\frac{b}{a^{5}}+\frac{a}{b^{5}}+\frac{v}{a^{3} b}+\frac{v}{a b^{3}}\right)$, and $D=\frac{2 E h^{3}}{3\left(1-v^{2}\right)}$

The values obtained are inserted in equation (5):

$$
\begin{aligned}
& \frac{\delta}{\delta a_{1}}\left(\frac{\mu h \omega^{2} a b}{4} a_{1}^{2}-\frac{\pi^{4} D K_{1}}{4} a_{1}^{2}-i \gamma_{0} \frac{\pi^{4} D K_{1}}{4} a_{1}^{2}-\right. \\
& \left.-i \gamma_{1} \frac{\pi^{4} D h K_{2}}{3} a_{1}^{3}+F_{0} a_{1}\right)=0 .
\end{aligned}
$$

By solving the equation, we obtain

$$
a_{1}=\frac{H}{\left(1-\frac{\omega_{1}^{2}}{p_{1}^{2}}\right)+i\left(\gamma_{0}+\gamma_{1} \frac{2 h K_{2}}{K_{1}} a_{1}\right)},
$$

in which $p_{1}^{2}=\frac{\pi^{4} D K_{1}}{\mu h a b}$ and $H=\frac{2 F_{0}}{\pi^{4} D K_{1}}$.

By developing complex amplitude into the indicator form $a_{1}=a_{01} e^{i \omega t}$, we obtain the oscillation amplitude as follows:

$$
a_{01}=\frac{H}{\sqrt{\left(1-\frac{\omega_{1}^{2}}{p_{1}^{2}}\right)+\left(\gamma_{0}+\gamma_{1} \frac{2 h K_{2}}{K_{1}} a_{01}\right)^{2}}},
$$

It can be seen that the unknown amplitude $a_{01}$ is found in the right and in the left parts of the expression (8). Therefore, in this case, we have a non-linear equation that can be used to plot a resonance curve rather than a formula.

The vibration phase is the amplitude function:

$$
\varphi=\operatorname{arctg} \frac{-\left(\gamma_{0}+\gamma_{1} \frac{2 h K_{2}}{K_{1}} a_{01}\right)}{\left(1-\frac{\omega_{1}^{2}}{p_{1}^{2}}\right)},
$$

It can be clearly seen that a non-linear solution is obtained if at least two polynomial $\gamma=\gamma\left(\varepsilon_{0}\right)$ terms are taken.

Let us further consider a rectangular plate to compare linear and non-linear solutions quantitatively. For this plate $a=b, K_{1}=\frac{4}{a^{2}}, K_{2}=\frac{2(1+v)}{a^{4}}$,

$$
\alpha=\frac{2 h K_{2}}{K_{1}}=\frac{h(1+v)}{a^{2}} \text {, and } H=\frac{3 F_{0} a^{2}\left(1-v^{2}\right)}{4 \pi^{4} E h^{3}} .
$$

To plot a resonance curve, equation (8) is solved with respect to $\frac{\omega_{1}}{p_{1}}$ in the following way:

$$
\frac{\omega_{1}}{p_{1}}=\sqrt{1 \pm \sqrt{\frac{H^{2}}{a_{01}^{2}}-\left(\gamma_{0}+\alpha \gamma_{1} a_{01}\right)^{2}}}
$$

In case of resonance, when $\omega_{1}=p_{1}$, the following equation is obtained from (10):

$$
\alpha \gamma_{1} a_{01}^{2}+\gamma_{0} a_{01}-H=0 .
$$

The solution of the equation yields the resonance amplitude:

$$
a_{01 r e s}=\frac{-\gamma_{0} \pm \sqrt{\gamma_{0}^{2}+4 \alpha \gamma_{1} H}}{2 \alpha \gamma_{1}}
$$

In the case of a linear problem, $\gamma=\gamma_{0}$ and $\gamma_{1}=0$, and from the equation (11) we get

$$
a_{01 r e s}^{*}=\frac{H}{\gamma_{0}} \text {. }
$$

\section{Numerical examples}

Let us consider some particular data used in calculation: the material is steel $C m 3$ under static axial deformation and after 24-hour ageing, $E=2 \cdot 10^{5} \mathrm{Mpa}$, $v=0.3, \quad \gamma_{0}=1.27 \cdot 10^{-3}, \quad \gamma_{1}=3.2 ; \quad$ the plate is $a \times a=1.0 \times 1.0 \mathrm{~m}, h=5.0 \cdot 10^{-3} \mathrm{~m}$; the excitation force amplitude $F_{0}=10 \mathrm{~N}$ [12].

The calculation results are as follows:

$$
a_{01 r e s}=2.139, a_{01 r e s}^{*}=2.205, \Delta a_{01}=3.08 \% \text {, }
$$

i.e. a linear solution yields an error of $3.08 \%$.

To plot the resonance curves with a specified change, the resonance amplitude is decreased and the respective values for the relationship $\frac{\omega_{1}}{p_{1}}$ are calculated by using formula (10). In a linear problem, $\gamma_{1}=0$ is assumed in formula (10).

The data obtained in the calculation are presented in table 1 and graphically shown in figure 1 . 
Table 1. Resonance curve calculation data

\begin{tabular}{|l|c|c|c|c|c|c|c|}
\hline \multicolumn{7}{|c|}{$\gamma=\gamma_{0}+\gamma_{1} \varepsilon_{1}$} \\
\hline $\begin{array}{l}a_{01} \\
\mathrm{~mm}\end{array}$ & 2.205 & 2.139 & 1.6 & 1.2 & 0.8 & 0.4 & 0.1 \\
\cline { 2 - 9 }$\omega_{1}$ & - & 1.0 & 1.00059 & 1.00097 & 1.00163 & 1.00345 & 1.01394 \\
\hline$p_{1}$ & - & 1.0 & 0.99941 & 0.9990 & 0.99836 & 0.99654 & 0.98586 \\
\hline \multicolumn{7}{|c|}{$\gamma=\gamma_{0}$} \\
\hline$\omega_{1}$ & 1.0 & - & 1.0006 & 1.00098 & 1.00163 & 1.00341 & 1.01391 \\
\cline { 2 - 8 }$p_{1}$ & 1.0 & - & 0.99932 & 0.9990 & 0.99836 & 0.9965 & 0.9858 \\
\hline
\end{tabular}

The relationship between the resonance amplitudes and the excitation force amplitude was calculated by the formulas (12) and (13). The calculation results are presented in table 2 and graphically shown in figure 1.

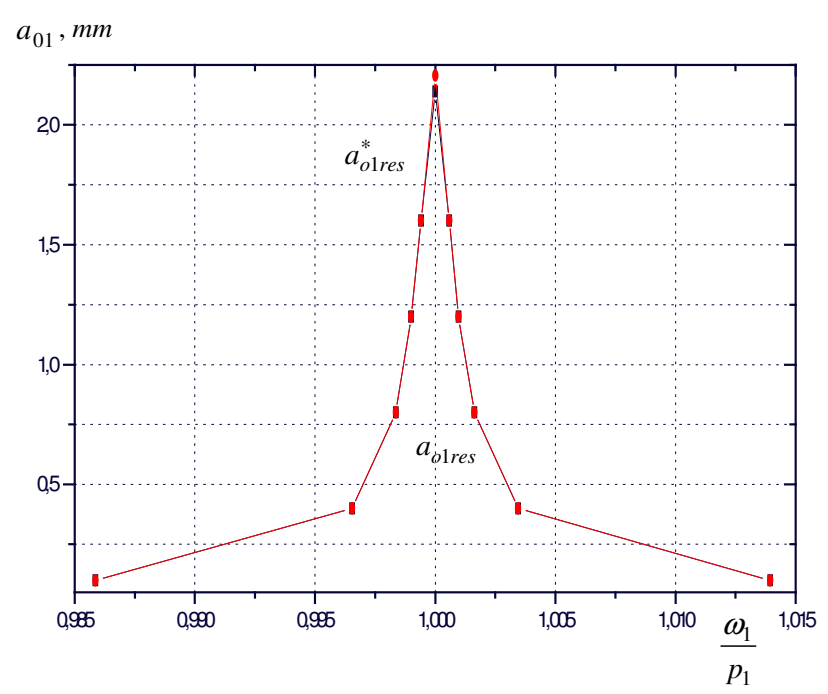

Fig 1. Graphs of resonance amplitudes: $a_{01 \text { res }}$, when

$$
\gamma=\gamma_{0}+\gamma_{1} \varepsilon_{0}, a_{01 \text { res }}^{*} \text {, when } \gamma=\gamma_{0}
$$

Table 2. The calculation data on the relationship between resonance amplitudes and excitation force

\begin{tabular}{|c|c|c|c|c|c|c|c|}
\hline$F_{0}, \mathrm{~N}$ & 0 & 5 & 10 & 15 & 20 & 25 & 30 \\
\hline $\begin{array}{c}a_{01 \text { res }}, \\
\mathrm{mm}\end{array}$ & 0 & 1.080 & 2.139 & 3.125 & 4.135 & 5.096 & 6.001 \\
\hline $\begin{array}{c}a_{01 \text { res }}^{*}, \\
\mathrm{~mm}\end{array}$ & 0 & 1.102 & 2.205 & 3.307 & 4.409 & 5.512 & 6.614 \\
\hline
\end{tabular}

\section{Conclusions}

The solution of a linear problem of plate oscillation yields a higher value and may be considered the first iteration of calculation.

When at least two terms of the polynomial $\gamma\left(\varepsilon_{0}\right)$ are taken, the resonance amplitude is decreased by more than 3 per cent.

The solution of the non-linear problem shows the dependence of the angle on the amplitude phase (8).
In the non-linear problem, as in the linear one, the relationship between resonance amplitude and excitation force is close to linear (Fig 2).

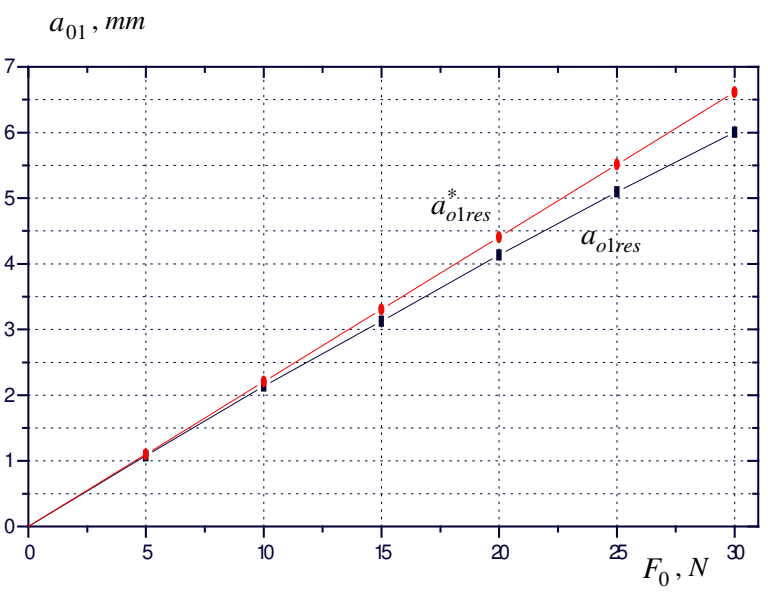

Fig 2. The relationship between resonance amplitudes and the excitation force

\section{References}

1. ALFANO, G., AURICCHIO, F., ROSATI, L. MITC. Finite elements for laminated composite plates. Methods Engng., 2001, no. 50, p. 707-738.

2. AURICCHIO, F., LOVADINA, C., SACCO, E. Analysis of mixed finite elements for laminated composite plates. Comput. Methods Appl. Mech. Engng., 2001, no. 190, p. 4767-4783.

3. BARADOKAS, P. Investigation of damping properties of composite construction. Modern Building Materials, Structures and Techniques. Vilnius: Technika, 1997, vol. 3, p. 112-116.

4. BARADOKAS, P., SYRUS, L. Dynamics of the plate with protective multi-coating. Plastics for the automotive industry (SP-1682). In SAE 2002 World Congress, Detroit, Michigan, 2002. 2002, p. 141145.

5. BARADOKAS, P., SYRUS, L. Forced oscillations of the plate with protective multi-coating. Aviation. Vilnius: Technika, 2002, vol. 6, p. 65-69. In Lithuanian.

6. BELEVIČIUS, R., RUSAKEVIČIUS, D. The optimization of the composite bending plates. Journal of Civil Engineering and Management. 2002, vol. 8, no. 4, p. 143-152. In Lithuanian.

7. BIRGER, IA., PANOKO, JG. Strength stability oscillation [Прочность, устойчивость, колебания]. Moscow: Mashynostrojenije, 1968, vol. 3, p. 567. In Russian.

8. CRISFIELD, MA. Non-linear finite element analysis of solids and structures. New York: John Wiley \& Sons, Ins., 1991, p. 345.

9. MICHNEVIČ, E., BELEVIČIUS, R. A laminated DKT element: modal analysis. Aviation. Vilnius: Technika, 1999, vol. 4, p. 94-99. In Lithuanian. 
10. NIKOLIC, Z., MICHANOVIC, A. Thin plate quadrilateral element with independent rotational DOF. Int. J. Engng. Modellin, 2003, vol. 16, no. 3, p. 256-269.

11. PILNER, R., JOSEPH, DS. A mixed finite element for plate bending with eight enhanced strain modes. Commun. Numer. Meth. Engng., 2001, no. 17, p. 443-454.

12. PISARENKO, GS., JAKOVLEV, AP., MATVEEV, VV. Characteristics of vibration attenuation of construction materials [Вибропоглощающие свойства конструкционных материалов). Kiev: Naukova Dumka, 1971, p. 357. In Russian.
13. SHEIKH, AH., HAIDAR, S., SENGUPTA, D. A high profession shear deformable element for the analysis of laminated composite plates of different shapes. Composite Structures, 2002, no. 55, p. 329336.

14. SOROKIN, ES. Dynamic calculation of bearing structure in buildings [Динамический расчет несущих конструкций зданий]. Moscow: Gosstroiizdat, 1956, p. 340. In Russian. 